\title{
XXV. Experiments for investigating the cause of the coloured concentric rings, discovered by Sir Isaac Newton, between two Object-glasses laid upon one another
}

\author{
William Herschel LLD. F.R.S.
}

To cite this article: William Herschel LLD. F.R.S. (1808) XXV. Experiments for investigating the cause of the coloured concentric rings, discovered by Sir Isaac Newton, between two Object-glasses laid upon one another, Philosophical Magazine Series 1, 30:118, 115-128, DOI: 10.1080/14786440808563784

To link to this article: http://dx.doi.org/10.1080/14786440808563784

$$
\text { 曲 Published online: } 18 \text { May } 2009 .
$$

Submit your article to this journal ¿

\section{山ll Article views: 2}

Q View related articles ¿ 
jected like the common clyster with sufficient force; otherwise oiled leathers should he nicely put round the joints of the machine, and a wet cloth round the injecting pipe, to prevent the return of the smoke by the sides of it. Clysters of carbonated hydrogen gas, or of other factitious airs, might be tried.

"Harrowgate water taken into the stomach, so as to induce six or seven stools every morning, for four or six weeks, is perbaps the most efficacious method in common use. A factitious Harrowgate water may be made probably of greater efficacy than the natural, by dissolving one ounce of marine salt, (called bay salt,) and half an ounce of magnesia Glauber's salt, (called Epsom salt, or bitter purging salt,) in twenty-eight ounces of water. A quarter or half a pint of this is to be taken every hour or two hours in the morning, till it operates, with a tea-spoonful of a solution of liver of sulphur, which is to be made by putting an ounce of hepar sulphuris into half a pint of water."

[To be continued.]

XXV. Experiments for investigating the Cause of the coloured concentric Rings, discovered by Sir IsAAC NewTon, letween two Olject-glasses laid upon one another. By William Herschel, LLD. F.R.S.

[Continued from p. 90.]

XV. Of the sudden Change of the Size and Colour of the Rings in different Sets.

$W_{\text {Hen two sets of rings are viewed which are dependent }}$ upon each other, the colour of their centres and of all the rings in each set, may be made to undergo a sudden change by the approach of the shadow of the point of a penknife or other opake slender body. To view this phænomenon properly, let a 16 -inch double convex lens be laid upon a piece of looking-glass, and when the contact between them has been made to give the primary set with a black centre, that of the secondary will be white. To keep the lens in this 
contact, a pretty heavy plate of lead with a circular hole in it of nearly the diameter of the lens should be laid upon it. The margin of the hole must be tapering, that no obstruction may be made to either the incident or reflected light. When this is properly arranged, bring the third shadow of the penknife upon the primary set, which is that towards the light. The real colours of this and the secondary set will then be seen to the greatest advantage. When the third shadow is advanced till it covers the second set, the second shadow will at the same time fall upon the first set, and the colour of the centres, and of all the rings in both sets, will undergo a sudden transformation from black to white and white to black.

The alternation of the colour is accompanied with a change of size, for as the white rings before the change were of a different diameter from the black ones, these latter, having now assumed a black colour, will be of a different size from the former black ones.

When the weight is taken from the lens the black contact will be changed into some other. In the present experiment it happened that the primary set got an orangecoloured centre, and the secondary a green one. The same way of proceeding with the direction of the shadow being then pursued, the orange centre was instantly changed to a green one, while at the same moment the green centre was turned into orange. With a different contact I have had the primary set with a blue centre and the secondary with a deep yellow one; and by bringing the second and third shadows alternately over the primary set, the blue centre was changed to a yellow, and the yellow centre to a blue one; and all the rings of both sets had their share in the transformation of colour and size.

If there are three sets of rings, and the primary set has a black centre, the other two will have a white one; and when the lowest shadow is made to fall on the third set, the central colour of all the three sets will be suddenly changed, the first from black to white, the other two from white to black.

A full explanation of these changes, which at first sight 
have the appearance of a magical delusion, will be found in a future article.

XVI. Of the Course of the Rays by which different Sets of Rings are seen.

In order to determine the course of the rays, which give the rings both by reflection and by transmission, we should begin from the place whence the light proceeds that forms them. In figure 1, (Pl.VI.) we have a plano-convex lens laid upon three slips of glass, under which a metalline mirror is placed. An incident ray, 1, 2, is transmitted through the first and second surface of the lens, and comes to the point of contact at 3. Here the rings are formed, and are both reflected and transmitted: they are refiected from the upper surface of the first slip, and pass from 3 to the eye at $4:$ they are also transmitted through the first slip of glass from 3 to 5 ; and at 5 they are again both reflected and transmitted; reflected from 5 to 6 , and transmitted from 5 to 7 ; from 7 they are reflected to 8 , and transmitted to 9 ; and lastly they are reflected from 9 to 10 . And thus four complete sets of rings will be seen at $4,6,8$, and 10 .

The most convenient way of viewing the same rings by transmission, is that which has been mentioned in the second article of this paper, when light is conveyed upwards by reflection. In figure 2 , consisting of the same arrangement of glasses as before, the light by which the rings are to be seen comes either from 1,2 , or 3 , or from all these places together, and being reflected at 4,5 , and 6 , rises up by transmission to the point of contact at 7 , where the rings are formed. Here they are both transmitted up to the eve at 8 , and reflected down to 9 ; from 9 they are refiected up to 10 , and transmitted down to 11 ; from 11 they are reficcted to 12, and transmitted to 13 ; and lastly, from 13 they are reflected to 14 ; so that again four sets of rings will be seen at $8,10,12$, and 14 .

This being a theoretical way of conceiving how the rays of light may produce the effects, it will be required to show by experiments that this is the actual progress of the rays, 
and that all the sets of rings we perceive are really reflected or transmitted in the manner that has been pointed out; but as we have so many reflections and transmissions before us, it will be necessary to confine these expressions to one particular signification when they are applied to a set of rings.

When the centre of the rings is seen at the point of contact, it is a primary set; and I call it reflected, when the rays which come to that point and form the rings undergo an immediate reflection. But I call it transmitted, when the rays after having formed the rings about the point of contact are inmediately transmitted.

Thus in figure 3 and 4 the rays $a b c, d e f$, give reflected sets of rings; and the rays $g h i, k l m$, in figure 5 and 6 , give transmitted sets:

In this denomination, no acconnt is taken of the course of the rays before they come to $a, d, g, k$; nor of what becomes of them after their arrival at $c, f, i, m$ : they may either come to those places or go from them by one or more transmissions or reflections, as the case may require; but our donomination will relate only to their course immediately after the formation of the rings between the glasses.

The secondary and other dependent sets will also be called reflected or transmitted by the same definition : and as a set of these rings formed originally by reflection may come to the eye by one or more subsequent transmissions; or being formed by transmission, may at last be seen by a reflection from some interposed surface, these subsequent transmissions or reflections are to be regarded only as convenient ways to get a good sight of them.

With this definition in view, and with the assistance of a principle which has already been proved by experiments, we may explain some very intricate phænomena ; and the satisfactory manner of accounting for them will establish the truth of the theory relating to the course of rays that has been described.

The principle to which I refer is, that when the pressure is such as to give a black centre to a set of rings seen by reflection, 
reflection, the centre of the same set, with the same pressure of the glasses, seen by transmission, will be white *.

I bave only mentioned black and white; but any other alternate colours, which the rings or centres of the two sets may assume, are included in the same predicament.

\section{Why two connected Sets of Rings are of alternate Colours.}

It has already been shown, when two sets of rings are seen, that their colours are alternate, and that the approach of the shadow of a penknife will cause a sudden change of them to take place. I shall now prove that this is a very obvious consequence of the course of rays that has been proposed. Let figure 7 and 8 represent the arrangement given in a precèding article, where a 16 -inch lens was laid upon a looking-glass, and gave two sets of rings with centres of different colours: but let figure 7 give them by one set of rays, and figure 8 by another. Then, if the incident rays come in the direction which is represented in figure 7 , it is evident that we see the primary set with its centre at 2 by reffection, and the secondary one at 4 by transmission. Hence it follows, in consequence of the admitted principle, that if the contact is such as to give us the primary set with a black centre, the secondary set must have a white one; and thus the reason of the alteriation is explained.

But if the rays come as represented in figure 8 , we see the primary set by transmission, and the secondary one by reflection; therefore, with an equal pressure of the glasses, the primary centre must now be white, and the secondary one black.

Without being well acquainted with this double course of rays, we shall be liable to frcquent mistakes in our estimation of the colour of the centres of two sets of rings ; for by a certain position of the light, or of the eye, we may see ont set by one light and the other set by the other.

XVIII. Of the Cause of the sudden Change of the Colours. Having thus accounted for the ahemation of the ceistral

* See Article XI. of this

II 4

colours, 
colours, we may easily conceive that the interposition of the penknife must have an instantaneous effect npon them. When it stops the rays of figure 7, which will happen when its second shadow falls upon the primary set, the rings will then be seen by the rays $1,2,3,4$, and $1,2,3,5,6$, of figure 8 . When it stops the rays of figure 8 , which must happen when the third shadow falls upon the primary set, we then see both sets by the rays $1,2,3$, and $1,2,4,5$, of figure 7 . When the penknife is quite removed both sets of rays will come to the point of contact, and in some respects interfere with each other; but the strongest of the two, which is generally the direct light of figure 7 , will prevail. This affords a complete explanation of all the observed phænomena: by the rays of figure 7 the centres will be black and white; by those of figure 8 they will be white and black; and by both we shall not see the first set so well as when the third shadow being upon it has taken away the rays of figure 8 : indeed we can hardly see the secondary set at all, till the shadow of the penknife has covered either the rays of figure 7 or of figure 8 .

As soon as we are a little practised in the management of the rays, by knowing their course, we may change the colour so gradually as to have balf the centre white while the other half shall still remain black; and the same may be done with green and orange, or blue and yellow centres. The rings of both sets will also participate in the gradual change; and thus what has been said of the course of rays in the 16 th article will again be confirmed.

XIX. Of the Place where the different Sets of Rings are to. le seen.

By an application of the same course of the rays, we may now also detcrmine the situation of the place where the different sets of rings are seen: for according to what has been said in the foregoing article, the situation of the primary set should be between the lens and the surface of the lookingglass: and the place of the secondary one at the metalline coating of the lowest surface. To try whether this be actually as represented, let us substitute a metalline mirror with 
with a slip of glass laid upon it in the room of the piece of looking-glass; and let there be interposed a short bit of wood, one-tenth of an inch thick, between the slip of glass and the mirror, so as to keep up that end of the slip which is towards the light. This arrangement is represented in figure 9 , where both sets of rays are delineated. Then if we interpose a narrow tapering strip of card, discoloured with japan ink, between the slip of glass and the mirror, so as to cover it at 7 , we do not only still perceive the primary set, but see it better than before: which proves that, being situated above the slip of glass, the card below cannot cover it. If on the contrary we insert the strip of card far enough, that it may at the same time cover the mirror both at 4 and at 7 , we shall lose the secondary set; which proves that its situation was on the face of the mirror.

When several sets of rings are to be perceived by the same eye-glass, and they are placed at different distances, a particular adjustment of it will be required for each set, in order to see it well defined. This will be very sensible when we attempt to see three or four sets, each of them situated lower than the preceding; for without a previous adjustment to the distance of the set intended to be viewed we shall be seldom successful : and this is therefore a corroborating proof of the situation that has been assigned to different sets of rings.

\section{Of the Connection between different Sets of Rings.}

It will now be easy to explain in what manner different sets of rings are connected, and why they have been called primary and dependent. When the incident rays come to the point of contact and form a set of rings, I call it a primary one: when this is formed some of the same rays are continued by transmission or reflection, but modified so as to convey an image of the primary set with opposite colours forward through any number of successive transmissions or reflections: whenever this image comes to the eye, a set of rings will again be seen, which is a dependent one. Many proofs of the dependency of second, third, and fourth 
scts of rings upon their primary one may be given ; - I shall only mention a few.

When two of rings are seen by a lens placed upon a looking-glass, the centre of the secondary set will always remain in the same plane with the incident and reflected rays passing through the centre of the primary one. If the point of contact, by tilting is changed, the secondary set will follow the motion of the primary set; and if the looking-glass is turned about, the secondary will be made to describe a circle upon that part of the looking-glass which surrounds the primaty one as a centre. If there is a defect in the centre or in the rings of the primary set, there will be exactly the same defect in the secondary one; and if the rays that cause the primary set are eclipsed, both sets will be lost together. If the colour of the primary one is changed, that of the secondary will also undergo its alternate change, and the same thing will hold good of all the dependent rings when three or four sets of them are seen that have the same primary one.

The dependency of all the sets on their primary one may also be perceived when we change the obliquity of the incident light; for the centres of the rings will recede from one another when that is increased and draw together when we lessen it, which may go so far that by an incidence nearly perpendicular we shall bring the dependent sets of rings almost under the prinary one.

XXI. To account for the Appearance of several Sets of Rings with the same coloured Centres.

It has often happened that the colour of the centres of different sets was not what the theory of the alternation of the central colours would have induced me to expect: I have seen two, three, and even four sets of rings, all of which had a white centre. We are however now sufficiently prepared to account for every appearance selating to the colour of rings and their centres.-

Let an arrangement of glasses be as in figure 9. When this is laid down so as to receive an illumination of daylight, 
light, which should not be strong, nor should it be very oblique, the reflection from the mirror $\mathrm{ill}$ then exceed that from the surface of glass; therefore the primary set will be seen by the rays 6,7 , coming to the mirror at 7 , and going through the point of contact in the direction $7,2,3$; which proves it to be a set that is seen by transmission, and it will therefore have a white ceritre. The rays 1, 2, 4, passing through the point of contact, will also form a transmitted set with a white centre, which will be seen when the reflection from 4 to 5 conveys it to the eye. But these two sets have no connection with each other; and as primary sets are independent of all other sets, I have only to prove that this secondary set belongs not to the primary one which is seen, but to another iuvisible one. This may be done as follows :

Introduce the black strip of card that has been mentioned before, till it covers the mirror at 7 ; this will take away the strong reflection of light which overpowers the feeble illumination of the rays 1,2, 3; and the real hitherto eclipsed primary set belonging to the secondary one with a white centre, will instantly make its appearance with a black one, We may alternately withdraw and introduce again the strip of card, and the centre of the primary set will be as often changed from one colour to its opposite, but the secondary set, not being dependent on the rays 6,7 , will not be in the least affected by the change.

If the contact should have been such as to give both sets with orange centres, the introduction of the strip of card will prove that the set which is primary to the other bas really a green centre.

Another way of destroying the illusion is to expose the same arrangement to a brighter light, and at the same time to increase the obliquity of the angle of incidence : this will give a sufficient reflection from the surface of the glass to be no longer subject to the former deceptive appearance; for now the centre of the primary set will be black, as it ought to be. 
XXII. Of the reflecting Surfaces.

The rays of light that form rings between glasses must undergo certain modifications by some of the surfaces through which they pass, or from which they are reflected; and to find out the nature of these modifications, it will be necessary to examine which surfaces are efficient. As we see rings by reflection and also by transmission, I shall begin with the most simple, and show experimentally the situation of the surface that reflects, not only the primary, but also the secondary sets of rings.

Upon a slip of glass, the lowest surface of which was deprived of its polish by emery, I laid an object-glass of 21 feet focal length, and saw a very complete set of rings. I then put the same glass upon a plain metalline mirror, and saw likewise a set of them. They were consequently not reflected from the lowest surface of the subjacent glass or metal.

It will easily be understood, that were we to lay the same object-glass upon a slip of glass emeried on both sides, or upon an unpolished metal, no rings would be seen. It is therefore neither from the first surface of the incumbent object-glass, nor from its lowest, that they are reflected; for if they could be formed without the modification of reflection from the upper surface of a subjacent glass or metal, they would still be seen when laid on rough surfaces; and consequently, the efficient reflecting surface, by which we see primary sets of rings, is that which is immediately under the point of contact.

To see a secendary set of rings by reflection, is only an inversion of the method of seeing a primary one. For instance, when a lens is laid upon a looking-glass, the course of the rays represented in figure 8 , will show that the rays $1,2,3,5,6$, by which a secondary set is seen, are reflected about the point of contact at 3 , and that the lowest surface of the incumbent lens is therefore the efficient reflecting one; and thus it is proved, that in either case of seeing reflected rings, one of the surfaces that are joined at the point of contact contributes to their formation by a certain modi. fication of reflection. 


\section{Of the transmitting Surfaces.}

It would seem to be almost self-evident, that when a set of rings is seen by transmission, the light which occasions them must come through all the four surfaces of the two glasses which are employed; and yet it may be shown that this is not necessary. We mav, for instance, convey light into the body of the subjacent glass through its first surface, and let it be reflected within the glass at a proper angle, so that it may come up through the point of contact, and reach the eye, having been transmitted through no more than three surfaces. To prove this I used a small box, blackened on the inside, and covered 'with a piece of black pasteboard, which had a hole of about half an inch in the middle. Over this hole I laid a slip of glass with a 56 -inch lens upon it; and viewed a set of rings given by this arrangement very obliquely, that the reflection from the slip of glass might be copious. Then guarding the point of contact between the lens and the slip of glass from the direct incident light, I saw the rings, after the colour of their centre had been changed, by means of an internal reflection from the lowest surface of the slip of glass; by which it rose up through the point of contact, and formed the primary set of rings, without having been transmitted through the lowest surface of the subjacent glass. The number of transmitted surfaces is therefore by this experiment reduced to three; but $I$ shall soon have an opportunity of showing that so many are not required for the purpose of forming the rings.

XXIV. Of the Action of the first Surface.

We have already shown that two sets of rings may be seen by using a lens laid upon a slip of glass; in which case, therefore, whether we see the rings by reflection or by transmission, no more than four surfaces can be essential to their formation. In the following experinents for investigating the action of these surfaces I have preferred metalline reflection, when glass was not required, that the apparatus might be more simple.

Upon a plain metalline mirsor I laid a double convex lens, having 
having a strong emery scratch on its upper surface. When I saw the rings through the scratch, they appeared to have a black mark across them. By tilting the lens, I brought the centre of the rings upon the projection of the scratch, so that the incident light was obliged to come through the scratch to the rings, and the black mark was again visible upon them, but much stronger than before. In neither of the situations were the rings disfigured. The stronger mark was owing to the interception of the incident light; but when the rings had received their full illumination the mark was weaker, because in the latter case the rings themselves were probably complete, but in the former deficient.

I placed a lens that had a very scabrous polish on one side, but was highly polished on the other, upon a metalline mirror. The defective side being uppermost, I did not find that its scabrousness had any distorting effect upon the rings.

I splintered off the edge of a plain slip of glass; it broke as it usually does with a waving striated, curved slope coming to an edge. The splintered part was placed upun a convex metalline mirror of 2 -inch focus, as in figure 10 . The irregularity of the striated surface through which the incident ray 1, 2, was made to pass had very little effect upon the form of the rings ; the strix appearing only like fine dark lines, with hardly any visible distortion; but when, by tilting, the returning ray, 2, 3, was also brought over the striated surface, the rings were much disfigured. This experiment therefore seems to prove that a very regular refraction of light by the first surface is not necessary ; for though the rings were much disfigured when the returning light came through the splintered defect, this is no more than what must happen to the appearance of every object which is seen through a distorting medium.

I laid the convex side of a plano-convex lens $2 \cdot 8$-inch focus with a diameter of 1.5 upon a plain mirror, and when J saw a set of rings I tilted the lens so as to bring the point of contact to the very edge of the lens, both towards the light and from the light, which, on account of the large diameter of the lens, gave a great varicty' in the angle of incidence to the rays which formed the rings; but no diffe- 
rence in their size or appearance could be perceived. This seems to prove that no modification of the first surface in which the angle of incidence is concerned, such as refraction and dispersion, has any share in the production of the rings, and that it acts merely by the intromission of light; and though even this is not without being influenced by a change of the angle, it can only produce a small difference in the brightness of the rings.

A more forcible argument, that leads to the same conclusion, is as follows: Laying down three 54-inch double convex leuses, I placed upon the first the plain side of a plano-convex lens of $\frac{5}{8}$ inch focus; upon the second, a plain slip of glass; and upon the third, the plain side of a plano-concave lens also $\frac{5}{8}$ inch focus. I had before tried the same experiment with glasses of a greater focal length, but selected these to strengthen the argument. Then, as nothing could be more different than the refraction of the upper surfaces of these glasses, I examined the three sets of rings that were formed by these three combinations, and found them so perfectly alike that it was not possible to perceive any difference in their size and colour. This shows that the first surface of the incumbent glasses merely acts as an inlet to the rays that afterwards form the rings.

To confirm the idea that the mere admission of light would be sufficient, $I$ used a slip of glass polished on one side but roughened with emery on the other: this being laid upon a 21 -feet object-glass, I saw a set of rings through the rough surface; and though thcy appeared hazy, they were otherwise complete in figure and colour. The slip of glass when laid in the same manner upon the letters of a book made them appear equally hazy; so that the rings were probably as sharply formed as the letters.

Having now already great reason to believe that no modification, that can be given by the first surface to the incident rays of light, is essential to the formation of the rings, I made the following decisive experiment:

Upon a small piece of looking-glass I laid half a double convex lens of 16 -inches focus, with the fracture exposed to the light, as represented in figure 11 . Under the edge of 
the perfect part of the lens was put a small lump of wax; soft enough to allow a gentle pressure to bring the point of contact towards the fractured edge, and to keep it there. In this arrangement it has already been shown that there are two different ways of seeing two sets of rings: by the rays $1,2,3$, we see a primary set; and by $1,2,4,5$, the secondary set belonging to it: by the rays $6,7,2,3$, we see a different primary set; and by $6,7,2,4,5$, we see its secondary one. That this theory is well founded has already been proved; but if we should have a doubt remaining, the interposition of any small opaque object upon the lookingglass near the fracture will instantly stop the latter two sets of rings, and show the alternate colours of the two sets that will then be seen by the rays $1,2,3$, and $1,2,4,5$. Remove in the next place the stop from the looking-glass, and bring the second shadow of the penknife over the primary set, and there will then only remain the two sets of rings formed by incident rays which come from 6 , and which have never passed through the upper surface of the lens. Now, as both sets of rings in this case are completely formed by rays transmitted upwards from the coated part of the looking-glass without passing through the first surface of the incumbent lens, the proof that the modifying power of that surface is not required to the formation of the rings is established.

It can hardly be supposed that the frrst surface of the lens should have any concern in the formation of the rings when the rays are reflected from the looking-glass towards the eye; but the same experiment, that has proved that this surface was not required to be used with incident rays, will show that we may do without it when they are on their return. We need only invert the fractured lens, as in figure 12, when either the rays $1,2,4,5$, or $6,7,2,4,5$, will convey the image of the rings after their formation to the eye without passing through any part of the lens.

[To be continued.] 